\title{
Study on an Interactive System for Conceptual and Basic Design of Machine Tool Structure
}

\author{
Zhangyong $\mathrm{YU}^{1}$, Keiichi NAKAMOTO ${ }^{1}$, Tohru ISHIDA $^{1}$, and Yoshimi TAKEUCHI ${ }^{1}$ \\ ${ }^{1}$ Department of Mechanical Engineering, Graduate School of Engineering, Osaka University \\ 2-1 Yamada-oka, Suita, Osaka 565-0871, Japan \\ E-mail: yu@cape.mech.eng.osaka-u.ac.jp
}

\begin{abstract}
:
This paper presents an improvement of design efficiency of machine tool structure in the stage of conceptual and basic design. An interactive system has been developed for designing machine tool structure. The distinguishing point in this system is that several conceptual beams are used to compose the total structure of machine tool because it is easy to analyze the static, dynamic and thermal properties. By converting the beam structure into an actual structure, the conceptual and basic design is completely finished. Consequently, the use of the interactive system allow, the designer to complete structural design of a machine tool in a shorter time with higher efficiency.
\end{abstract}

Keywords: Interactive system, Machine tool, Total structure, Conceptual and basic design, Structural design, Converting method

\section{Introduction}

In recent years, the static, dynamic and thermal stiffness of machine tool structure have improved. As a result, machining process achieves high speed, precision, and productivity dramatically. Nowadays, a lot of machine tool structures can be proposed in the stage of conceptual and basic design. Shinno and Ito et al. proposed the method that can create a lot of machine tool structures [1-7]. Moreover, a lot of new machine tool structures are created, based on designers' experiences. However, it takes long modeling and calculation time for these machine tool structures, thus leading to the difficult to select the most suitable structure in a short time, because the designer has to verify build the static, dynamic and thermal stiffness of machine tool structure. Though, Finite Element Analysis is very useful, it takes long time to build the analytic model and to calculate the characteristics. Therefore, it is necessary to develop a new method to reduce the time of conceptual and basic design and to help the designers.

The study presents an interactive system to efficiently accomplish structural design of machine tools. In the proposed system, the total machine tool structure is considered to consist of a lot of conceptual beams with the polar moment of inertia of area, the moment of inertia of area, the cross-sectional area, and the length. The freedom degrees of the total structure are small because each beam has only two endpoints and twelve freedoms, thus leading to easily establish or modify the matrix data, which are utilized to analyze static, dynamic, and thermal stiffness.

Firstly, the lengths of conceptual beams in machine tool structures are decided according to the design requirements. Then, in the static analysis stage, all of the moments are calculated, and all of the cross-sectional areas are considered as the same size because the influence caused by the cross-sectional areas is very small in this stage. In the dynamic analysis stage, all of the cross-sectional areas are calculated. After investigating the actual structures, the actual machine tool structures are formed from the structure consisting of conceptual beams depending on the polar moment of inertia of area, the moment of inertia of area, the cross-sectional area and the length. Finally, the appropriate structures are decided by analysis of the thermal stiffness.

By using the interactive system, a suitable machine tool structure can be selected, which has enough static, dynamic and thermal properties among several proposed structures. As a result, it is found that the system has the potential of utilizing as the design tool.

\section{Structure Design Method}

The structural design process in the study is divided into five steps: proposal, modeling, analysis, selection, and converting of conceptual and basic design to actual machine tool structure. Figure 1 shows the flowchart of these five steps. In the first step, machine tool structures are proposed to be composed of several beams. According to the requirements such as machining space, the original length of each beam can be determined. Of course, each structure has its special patterns, for example, the column is single type or double type. In the second step, mathematical models of each structure have to be established to analyze the static dynamic and thermal stiffness and to investigate the characteristics of each structure. The third step is analyzing the stiffness of all these structures that proposed in the first step. Then, 
in the forth step, the most suitable structure is selected, based on the analysis results. In the last step, a converting method has been proposed, which is used to convert the beam structure into the actual structure for realization of the integrity and practicality of this structural design method. Since the complex machine tool structure may be considered as a simple model, which composed of beams, it is easy to analyze the static, dynamic and thermal stiffness of beam structure. Therefore, the efficiency of structural design in the conceptual and basic stage is improved significantly.

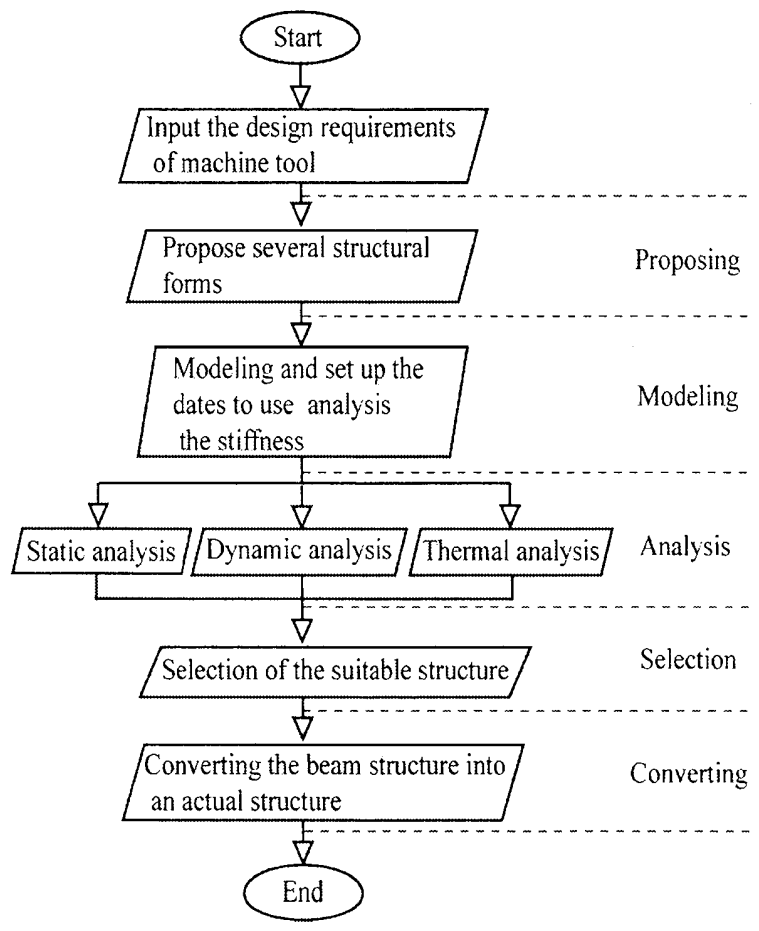

Figure 1: Flowchart of structural design.

\subsection{Proposing of structure types}

Requirements of machine tool design include three main aspects; production (machining capacity, high precision, high speed and so on), processing (shape and mass of work piece, etc), and environment (temperature humidity and vibration). According to the requirements, several structures can be proposed as candidates, based on designer's experience or by the method that proposed by Shinno and Ito et al. [1-7]. All these structures are considered to be composed of beams, and each beam serves as one part of machine tool. Therefore, according to the requirement of machining space, the length of each beam have to be determined preliminarily, and then the whole structure appears as the basic shape. During proposing of the structures, three principles have to be considered, that is, 1) same machining spaces, 2) same mass and 3) same material of each structure. The number of proposed structure is arbitrarily decided.

\subsection{Modeling of proposed structure types}

After proposing the structures, the next step is to establish mathematical model for investigating the static, dynamic, and thermal stiffness of proposed structures. Firstly, a Cartesian coordinate system on each structure is defined. Then, the coordinates of two endpoints of each beam can be determined, as shown in Fig. 2, where a machine tool structure is composed of beams. The structure has 13 endpoints and 14 beams. The points 1,5 , 9,10 are considered as fixed-points. The stiffness matrix $[\mathrm{k}]$ and the mass matrix $[\mathrm{m}]$ of each beam can be set on the basis of the beam theory. According to the combination of circumstance of the structure and by using the coordinate transform system, the total stiffness matrix $[\mathrm{K}]$ and the total mass matrix $[\mathrm{M}]$ can be also prepared. Parameters of the two matrixes include the cross-sectional area, polar moment of inertia of area, the moment of inertia of area, Young's modulus, Poisson's ratio, density, and coefficient of thermal expansion.

In order to investigate the thermal stiffness, it is required to calculate thermal deformations caused by the temperature change in the surrounding environment and a machining operation.

Cutting forces loaded on machine tool structure during a machining operation are complex. Thus, it is difficult to set the cutting forces accurately. In this study, the cutting forces are proposed as a constant value in the all-machining spaces.

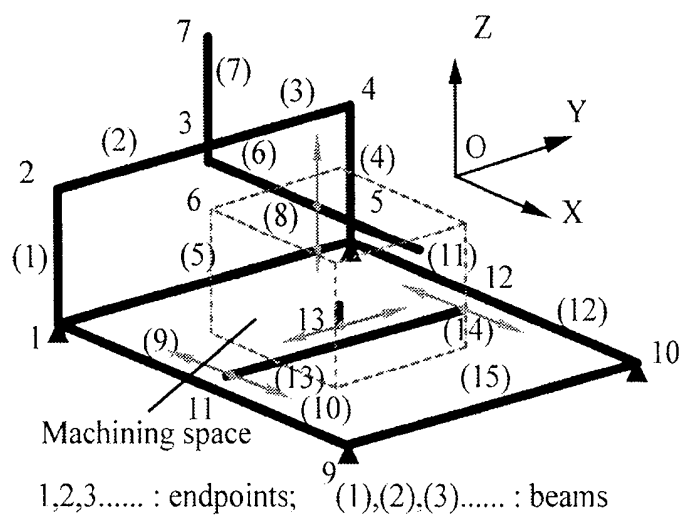

Figure 2: Beam model structure with numbers.

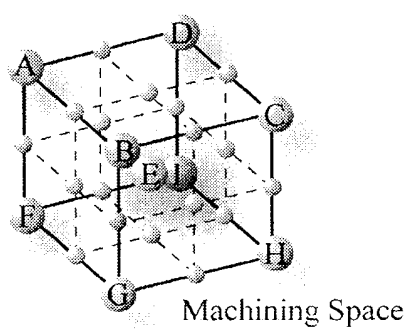

Figure 3: Analytic points in the machining space. 


\subsection{Analysis and selection suitable structure}

After determining the force $\{F\}$ loading on machine tool structure by the static analysis system, based on Eq. (1), the static displacement $\{x\}$ of the structure can be calculated. The natural frequencies can be calculated by another system, based on Eq. (2). The model of thermal conductivity in the study is simple because a one-dimensional heat conduction, Equation (3), allows to calculate the thermal displacements. It is impossible to analyze them at each point of the whole machining space because it has numerous points. Therefore, some typical points are selected. In general, twenty-seven points in the machining space are selected as analyzing locations. Among them, eight corner points and one center point, as shown in Fig. 3, are represent actives of the stiffness on the whole machining space.

Judging from the above three properties, the most suitable beam structure with good static, dynamic, and thermal stiffness can be selected.

$$
\begin{aligned}
& \{F\}=[K] \cdot\{x\} \\
& {[M]\{\ddot{x}\}+[K]\{x\}=\{0\}} \\
& \rho c A \frac{\partial T}{\partial t}+\lambda A \frac{\partial T}{\partial x} \\
& =-\lambda A \frac{\partial}{\partial x}\left(T+\frac{\partial T}{\partial x} d x\right)+\alpha s\left(T-T_{0}\right)
\end{aligned}
$$

$\rho \quad$ Density of the material

$C$ Specific of the material

A Cross-sectional area

$\lambda$ Traditional thermal conductivity

$\alpha \quad$ Coefficient of thermal traditional

$T$ Temperature at one point in the beam

$T_{0} \quad$ Surrounding environment temperature

$S$ Circumference of the cross-section of the beam

\subsection{Converting beam structure into actual structure}

Since the selected beam structure is not an actual structure, it is necessary to convert the beam structure into an actual structure. In order to obtain the ratio relation among the moment of inertia of area $I y$ and $I z$, the torsion constant $J$, and the cross-sectional area $A$ of actual structure cross-sections, the existing machine tool structures are investigated.

The underlying idea of the proposed converting method is to find proper ratio coefficients among the moment of inertia of area $I y$ and $I z$, the torsion constant $J$, and the cross-sectional area $A$, those of the beams composed the total structure. Figure 4 shows the flowchart of converting procedure. The first step is to find a ratio coefficient $\alpha_{i}$ among the beams. Then, ratio coefficient $\beta$, can be found among $I y, I z, J$ and $A$. The best ratio coefficients $I y_{i}, I z_{i}, J_{i}$ and $A_{i}$ are calculated by Eq. (4). According to the obtained ratio coefficients $I y_{i}$, $I z_{1}, J_{i}$ and $A_{i}$, the correlative part structure of the machine tool with a suitable cross-section shape can be selected.
Finally, according to the design requirements, the detailed size of the cross-section shape can be determined. Thereby, the converting from the beam structure into actual structure is realized.

$$
\left.\begin{array}{l}
I y_{i} \leftarrow I y_{i} \times \alpha_{i} \times \beta_{1} \\
I z_{i} \leftarrow I z_{i} \times \alpha_{i} \times \beta_{2} \\
J_{i} \leftarrow J_{i} \times \beta_{3} \\
A_{i} \leftarrow A_{i} \times \beta_{4}
\end{array}\right\}
$$

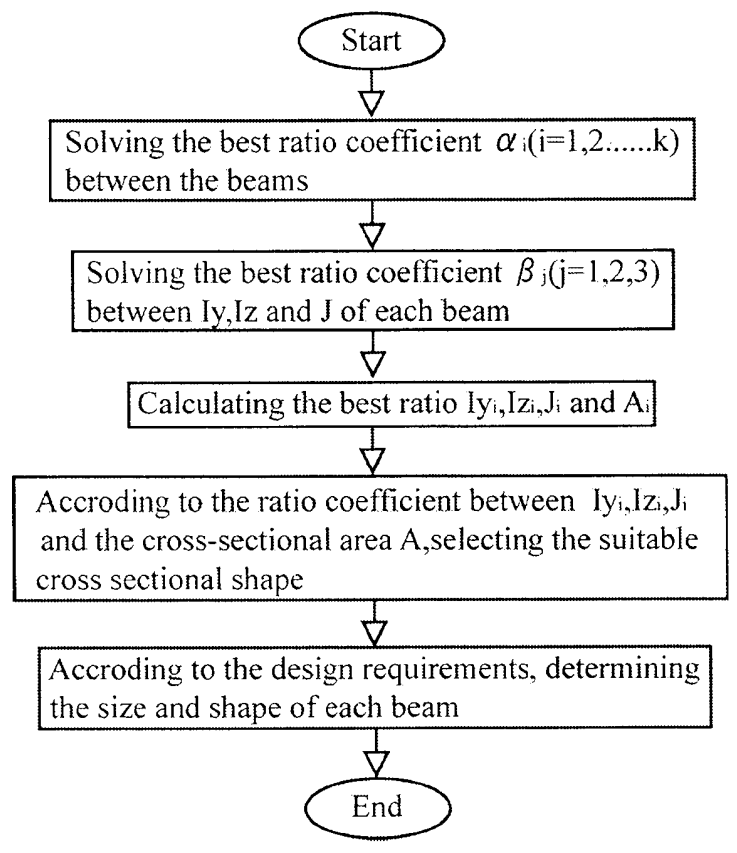

Figure 4: Flowchart of the converting procedure.

\section{Case Study of Conceptual and Basic Design}

Various machine tool structures can be proposed in the conceptual and basic design stage. In order to prove the usefulness of this interactive system, eight typically comparable beam structures, shown in Fig. 5, have been selected as the proposed original structures. The beam with arrows means movable parts of the structure. The movable direction and ranges such as $X, Y$, and $Z$ denoted by the arrows, stand for the machining space, which is represented cubic of each beam structure, as shown in the same figure. Each structure has the same machining space is $500 \times 500 \times 500 \mathrm{~mm}^{3}$. Assuring all the structures in the same mass, the cross-sectional areas are considered to have different sizes because the total length of each structure are different. The lengths and cross-sectional areas of these structures are listed in Table 1.

In addition, the same Yong's modulus $E: 2 \times 10^{11} \mathrm{~Pa}$, Poisson ratio $\gamma: 0.33$, density $\rho: 7850 \mathrm{~kg} / \mathrm{m}^{3}$, and linear expansion coefficient for each structure have been supposed. The cutting forces loading on machine tools are supposed to $F x: 1000 \mathrm{~N}, F y: 1000 \mathrm{~N}, F z: 1000 \mathrm{~N}$ for 
each structure. Moreover, in order to investigate the thermal stiffness of these structures, the surrounding temperature is proposed to change from $0{ }^{\circ} \mathrm{C}$ to $20^{\circ} \mathrm{C}$.
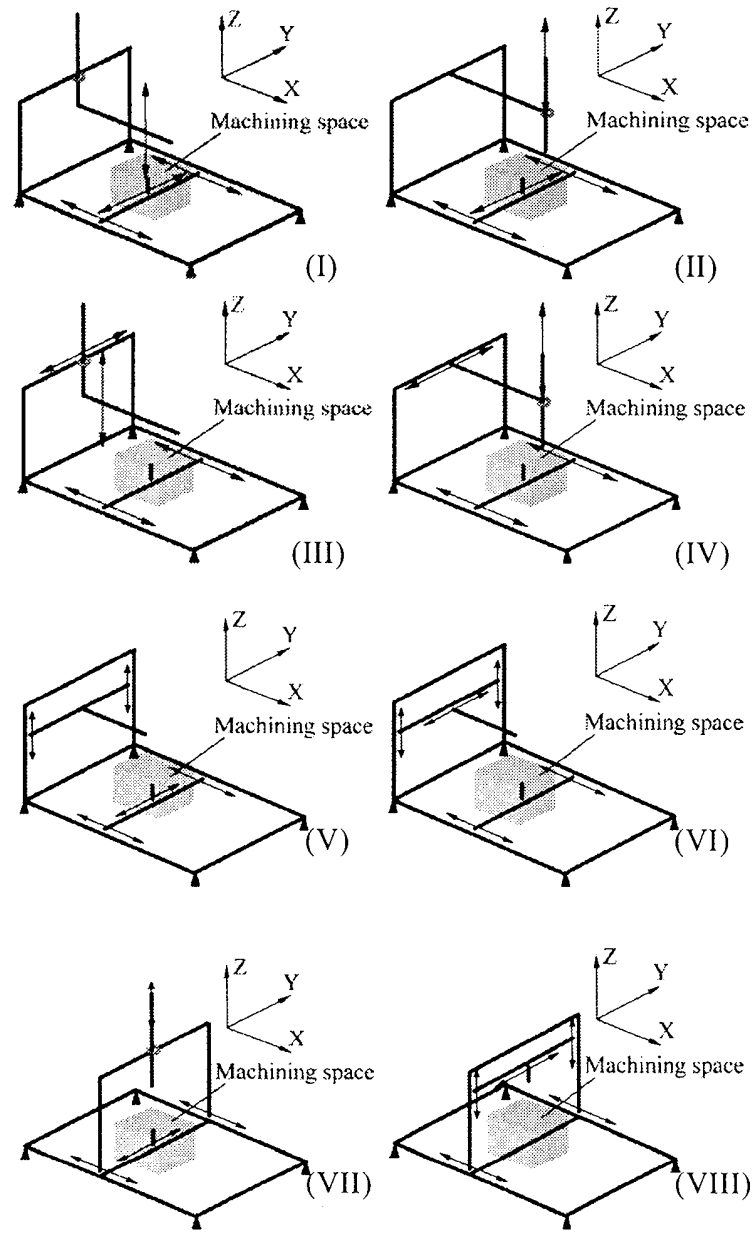

Figure 5: Beam structures to simulate.

Table 1: Total length of each structure

\begin{tabular}{c|c|c|c}
\hline Structure & Number of beam & Total length(mm) & Section $(\mathrm{mm} \times \mathrm{mm})$ \\
\hline I & 15 & 8000 & $100 \times 100$ \\
\hline II & 15 & 8000 & $100 \times 100$ \\
\hline III & 15 & 8000 & $100 \times 100$ \\
\hline IV & 15 & 8000 & $100 \times 100$ \\
\hline V & 16 & 8500 & $97.01 \times 97.01$ \\
\hline VI & 16 & 8500 & $103.3 \times 103.3$ \\
\hline VII & 16 & 7500 & $103.3 \times 103.3$ \\
\hline VIII & 17 & 9500 & $94.28 \times 94.28$
\end{tabular}

\section{Discussion for Case Study}

When the proposed cutting forces are loaded to these structures at one analyzing point, the static deformations take place. Figure 6 shows the example of static deformations of structure VIII. The obtained results show as error spaces of eight structures, as seen in Figure 7. It is obvious that the static stiffness of structure I, II, III, IV is weaker than structure V, VI, VII, VIII. Therefore, the four structures have been selected as candidates.
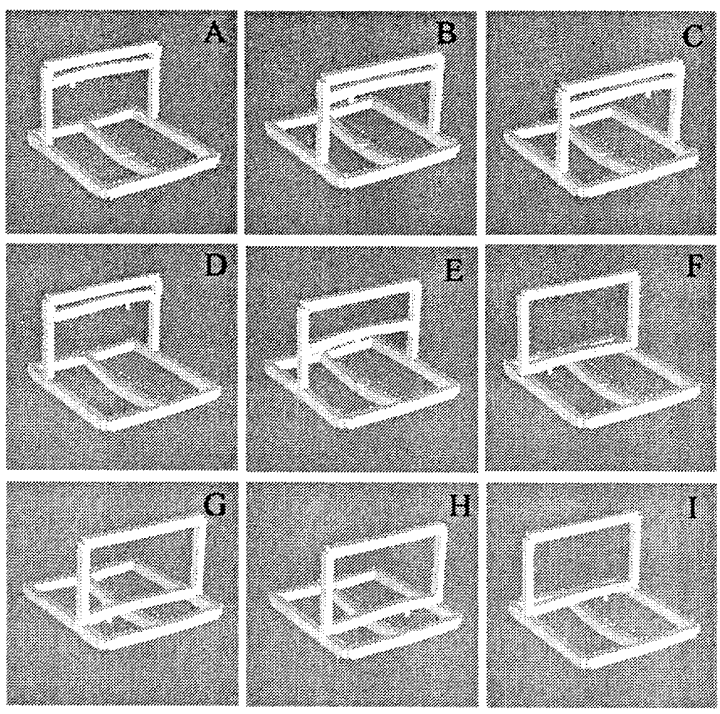

Figure 6: Static deformation of structure VIII

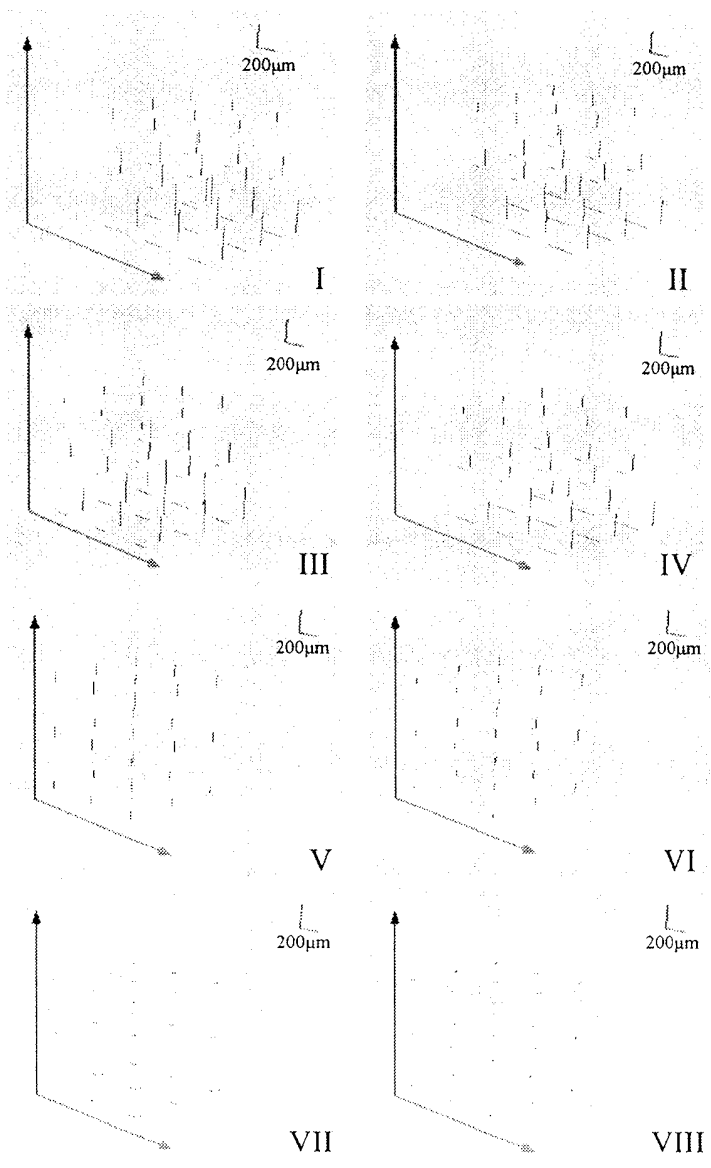

Figure 7: Error spaces of static deformation 
Natural frequency is an evaluation index of the structure dynamic stiffness. Thus, all these natural frequencies of eight structures are calculated, and the correlative dynamic deformation also is obtained. As an example, the parts of the dynamic deformations with their correlative natural frequency are shown in Fig. 8. Three lowest natural frequencies of eight structures are listed in Table 2. In general, the lowest natural frequency draws attention because the amplitude the lowest with this natural frequency is largest. Therefore, the structure V, VI and VIII have been selected as candidates because they have higher lowest natural frequency than others.

Table 2: Natural frequencies of each structure

\begin{tabular}{l|c|c|c|c|c|c|c|c|c}
\hline \multicolumn{2}{c|}{ Structure } & I & II & III & IV & V & VI & VII & VIII \\
\hline $\begin{array}{l}\text { Natural } \\
\text { frequency }\end{array}$ & Ist & 57.14 & 57.64 & 57.36 & 58.09 & 77.75 & 76.68 & 67.41 & 75.28 \\
\cline { 2 - 10 }$(\mathrm{Hz})$ & 2nd & 96.55 & 98.12 & 96.55 & 90.78 & 143.8 & 138.2 & 78.59 & 110.5 \\
\cline { 2 - 9 } & 3rd & 136.9 & 111.5 & 151.0 & 125.8 & 149.6 & 164.6 & 152.1 & 155.0 \\
\hline
\end{tabular}
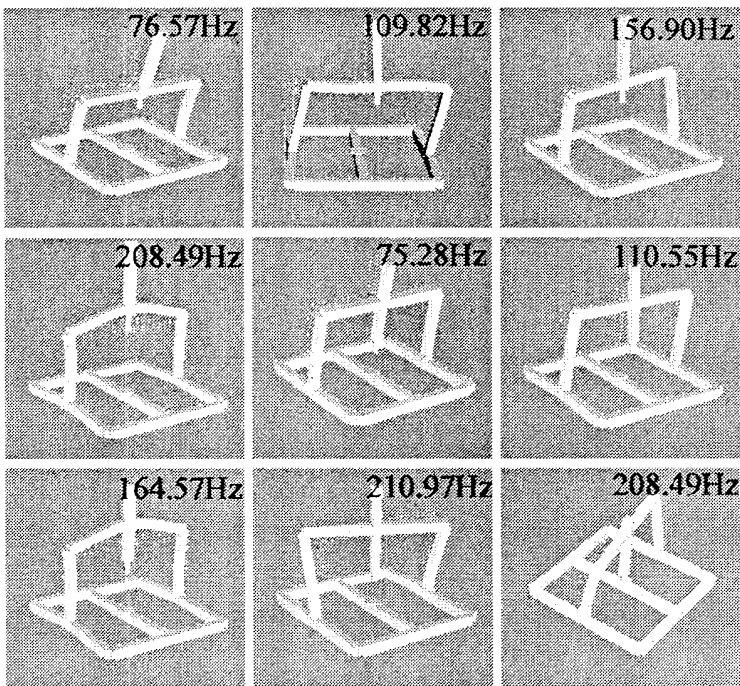

Figure 8: Dynamic deformation of structure VIIl

Thermal stiffness is another impartment evaluation index of machine tool structure, because using a machine tool throughout in a same surrounding temperature is impossible generally. Under the proposed changing temperature, thermal deformations of these eight structures are occurred. As an example, thermal deformations of structure VIII are shown in Fig. 9. All of the error spaces of these eight structures are shown in Fig 10. Thermal displacements of Structure I, II, III, IV, V, and VI are in the same level, and the displacements of structure VII and VIII are little small. Thus, the structure VII and VIII are selected as candidates for their higher thermal stiffness.

Considered in a comprehensive way, structure VIII is selected as a suitable structure among the eight proposed structures from the viewpoint of the static, dynamic and thermal stiffness. Certainly the selected structure VIII is a beam structure, then, method the actual structure can be obtained by using the converting.
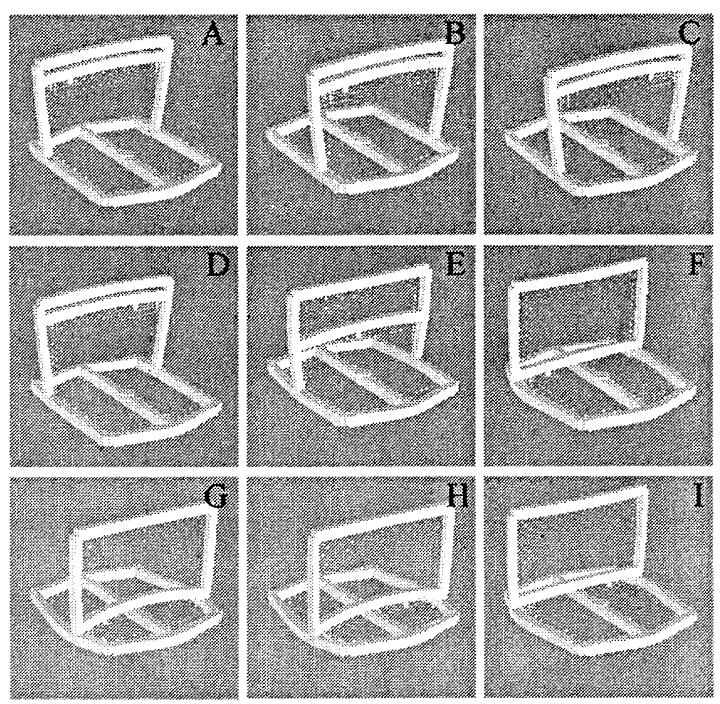

Figure 9: Thermal deformation of structure VIII
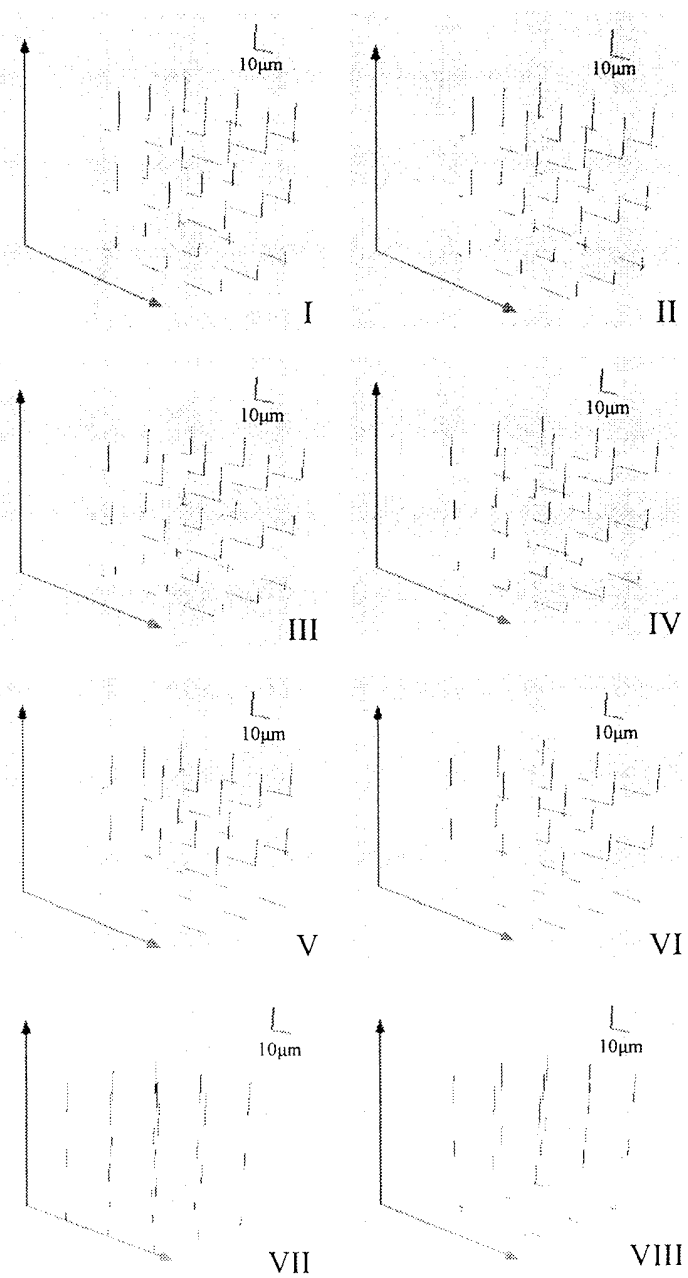

Figure 10: Error spaces of thermal deformation 


\section{Concluding Remarks}

This method is applicable to design a structure in the phase of conceptual and basic step of machine tool design. This design method, whose key idea is considered the total structure as a combination, which is composed of a finite number of concept beams, is presented to improve the effective design of machine tool structure. The whole design process does not build the huge date of the total structure that usually analyzes the stiffness of one structure, easily completing the total structure design. Therefore, a suitable structure can be designed in a short time. In other words, a preliminary of interactive system have been established. Simulation results show this method works more effectively in machine tool structure design, and have the potential to apply in other mechanism structure designs. The main results of the study are as follows:

1. The structure of machine tool is proposed to be composed of beams; the interactive system for conceptual and basic design of machine tool structure has been established. By proposed machine tool structure as beams, the whole analysis process is turned to easy, simple, and can be finished in a short time.

2. The analysis method of the static, dynamic, and thermal stiffness of the proposed machine tool structure is developed.

3. By assumption of eight structural patterns and analyzing their static, dynamic, and thermal stiffness, the most suitable structural pattern can be selected.

\section{References}

[1] Yoshimi ITO, Hidenori SHINNO, 1980, Structural Description of Machine Tools (1st report), Journal of the Japan Society of Mechanical Engineers, Vol.46, No.405(C series). pp562-571(in Japanese)

[2] Hidenori SHINNO, Yoshimi ITO, 1980, Structural Description of Machine Tools (2nd report), Journal of the Japan Society of Mechanical Engineers, Vol.46, No.405(C series). pp572-579(in Japanese)

[3] Hidenori SHINNO Yoshimi ITO, 1981, Structural Description of Machine Tools (3rd report), Journal of the Japan Society of Mechanical Engineers, Vol.48, No.425(C series). pp 124-130(in Japanese)

[4] H. SHINNO, Y. ITO: 1984, Generating Method for Structural Configuration of Machine Tools (1st report, Method of Variant Design Type), Journal of the Japan Society of Mechanical Engineers, Vol.50, No.449(C series). pp213-221(in Japanese)

[5] Hidenori SHINNO, Yoshimi ITO, 1984, Generating Method for Structural Configuration of Machine Tools (2nd report, Free Design Method), Journal of the Japan Society of Mechanical Engineers, Vol.50, No.459(C series). pp2192-2200(in Japanese)

[6] Hidenori SHINNO Yoshimi ITO, 1986, Generating Method for Structural Configuration of Machine tools (3rd report, Variant Design Using Directed
Graph), Journal of the Japan Society of Mechanical Engineers, Vol.52, No.474(C series). pp788-793(in Japanese)

[7] Yoshimi ITO, Hidenori SHINNO, 1982, Structural Description and Similarity Evaluation of The Structural Configuration in Machine Tools, Int.J.Mach.Tool Des.Res. Vol.22, No.2,pp97-110 